\title{
Portal Vein Flow by Doppler Ultrasonography and Liver Volume by Computed Tomography in Living Donor Candidates: Correlation with Indocyanine Green Test
}

\author{
Sunyoung Lee, ${ }^{1,2}$ Kyoung Won Kim, ${ }^{1}$ Jeongin Lee, ${ }^{3}$ Taeyong Park, ${ }^{3}$ \\ Gi-Won Song, ${ }^{4}$ Sung-Gyu Lee
}

\begin{abstract}
Objectives: Our goal was to investigate the correlation between indocyanine green test results and imaging parameters in living liver donor candidates.

Materials and Methods: Our study included 219 healthy donor candidates who were evaluated with Doppler ultrasonography (portal vein time average flow), computed tomography (liver volume), liver biopsy (fat fraction), and indocyanine green retention rate at 15 minutes.

Results: Portal vein time average flow $(r=-0.375)$, fat-free liver volume/body weight ratio $(r=-0.239)$, and portal vein time average flow $x$ fat-free liver volume/body weight ratio $(r=-0.424)$ showed significant correlations with indocyanine green retention rate at 15 minutes (all $P<.001$ ).

Conclusions: Imaging parameters were significantly correlated with indocyanine green retention rate at 15 minutes in living liver donor candidates.
\end{abstract}

Key words: Indocyanine green retention test, Liver transplantation, Liver volume-to-body weight ratio

\section{Introduction}

Indocyanine green (ICG) is a water-soluble, fluorescent tricarbocyanine dye that binds to plasma protein and is distributed in the blood. ${ }^{1}$ Extraction

From the ${ }^{1}$ Department of Radiology and Research Institute of Radiology and the ${ }^{4}$ Division of Liver Transplantation and Hepatobiliary Surgery, Department of Surgery, University of Ulsan College of Medicine, Asan Medical Center, Seoul, Korea; the 2Department of Radiology and Research Institute of Radiological Science, Severance Hospital, Yonsei University College of Medicine, Seoul, Korea; and the ${ }^{3}$ School of Computer Science \& Engineering, Soongsil University, Seoul, Korea

Acknowledgements: This research was supported by the Basic Science Research Program through the National Research Foundation (NRF) of Korea, funded by the Ministry of Science, ICT, and Future Planning (No. 2017R1E1A1A03070961). The authors have no conflicts of interest to declare.

Corresponding author: Kyoung Won Kim, Department of Radiology and Research Institute of Radiology, University of Ulsan College of Medicine, Asan Medical Center, 88 Olympic-ro 43-gil, Songpa-gu, Seoul 05505, Korea

Phone: +82 $230104400 \quad$ E-mail: kimkw@amc.seoul.kr

Experimental and Clinical Transplantation (2019) 5: 627-631 from the blood occurs exclusively by the liver, with selective uptake across the sinusoidal plasma membrane and removal via a carrier-mediated mechanism²; ICG is excreted into the bile without extrahepatic excretion or metabolism. ${ }^{3}$ The ICG test is most commonly used for dynamic quantitative assessment of liver function, especially in cases of major liver resection, including living-donor liver transplant.

A quantitative liver function test, including the ICG test, relies on the clearance principle, in which hepatic clearance is the product of liver blood flow and liver extraction capacity. ${ }^{2}$ Doppler ultrasonography is routinely used to preoperatively assess the vasculature in living-donor liver transplant and has been proven to be a reliable technique for estimating liver blood flow. ${ }^{4,5}$ Computed tomography (CT) is a standard technique to preoperatively evaluate liver volume in potential living donors. ${ }^{6}$ Functional liver volume reflects the liver extraction capacity in healthy individuals with normal hepatic function. ${ }^{2} \mathrm{We}$ hypothesized that, among healthy living donors, the ICG test could reflect portal vein (PV) flow as a component of liver blood flow by Doppler ultrasonography and functional liver volume by CT volumetry that excludes nonmetabolic liver tissue such as fat fraction.

The aim of our study was to investigate the correlation between ICG test results and imaging parameters, such as PV flow by Doppler ultrasonography and functional liver volume by CT volumetry, excluding nonmetabolic liver tissue (eg, fat fraction), in healthy donor candidates.

\section{Materials and Methods}

Study population

Our institutional review board approved this retrospective study and waived the requirement for 
informed consent. We consecutively included a total of 219 healthy donor candidates who were evaluated by Doppler ultrasonography, CT scan, percutaneous liver biopsy, and the ICG test between January 2011 and July 2011 to determine their suitability to serve as living liver donors. There were 136 men (mean age [ \pm standard deviation] of $28.6 \pm 8.9 \mathrm{y}$; range, 16-59 y) and 83 women (mean age of $33.3 \pm 10.5$ $\mathrm{y}$; range, 16-58 y), with an overall mean age of $30.4 \pm 9.8$ years (range, 16-59 y). The demographic data of the living donor candidates are summarized in Table 1.

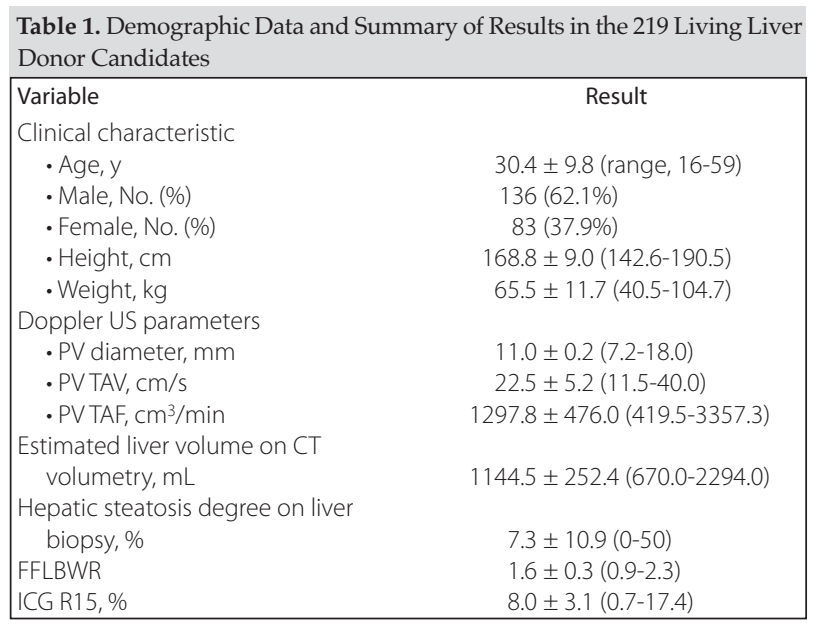

Abbreviations: $\mathrm{CT}$, computed tomography; FFLBWR, fat-free liver volumeto-body weight ratio; ICG R15, indocyanine green retention rate at 15 minutes; PV, portal vein; $\mathrm{TAF}$, time average flow; $\mathrm{TAV}$, time average velocity; US, ultrasonography

Unless otherwise indicated, results are means \pm standard deviations, with ranges in parentheses.

\section{Doppler ultrasonography techniques and analysis} Hepatic ultrasonographic examinations, including Doppler studies, were performed by board-certified abdominal radiologists using a Sequoia 512 machine (Acuson Siemens, Mountain View, CA, USA) equipped with a 1 - to $4-\mathrm{MHz}$ curved-array transducer. The main PV diameter was measured at the point from the porta hepatis to the right PV/left PV bifurcation. Standard Doppler parameters were measured by adjusting velocity scale and angle correction. The time average velocity of the PV (PV TAV, $\mathrm{cm} / \mathrm{s}$ ) was obtained within the center of the main PV where laminar flow is greatest, setting its dimension as wide as $\geq 50 \%$ of the vessel diameter, at the end of expiration with a spontaneous short breath-hold. The time average flow (TAF, $\mathrm{cm}^{3} / \mathrm{min}$ ) of the PV was calculated using the following equation: PV TAF $=\mathrm{PV}$ area $\left(\mathrm{cm}^{2}\right) \times \mathrm{PV}$
TAV $(\mathrm{cm} / \mathrm{s}) \times 60$ (where PV area was determined by $\left.\pi \times[\mathrm{PV} \text { diameter } / 2]^{2}\right)$. To minimize measurement error, all parameters were measured 3 times and were averaged for the analysis.

\section{Computed tomography scans and automated computed tomography volumetry}

Computed tomography scans were performed using 16-row multidetector CT scanners (Somatom Sensation 16; Siemens Medical Solutions, Erlangen, Germany). After unenhanced CT scans were obtained, we administered $150 \mathrm{~mL}$ of iopromide (Ultravist 370; Bayer Schering, Berlin, Germany) at a flow rate of $3 \mathrm{~mL} / \mathrm{s}$ using a mechanical injector (Percupump II; E-Z-Em, Westbury, NY, USA). Biphasic CT images were obtained during the hepatic arterial phase (HAP) and portal venous phase (PVP). By using bolus-tracking methods (CARE-Bolus, Siemens Medical Solutions), HAP scanning was initiated 10 seconds after the enhancement of the descending aorta reached 100 HU; PVP scanning was initiated 40 seconds after HAP scanning.

We used computer-aided hepatic volumetry on PVP CT images. The initial hepatic outline was detected through the sequential application of seeded region growing onto level-set speed images, which were generated as a map inversely proportional to the gradient magnitude. ${ }^{7}$ The Malladi level-set method ${ }^{8}$ was adopted for level-set propagation. A rolling ball algorithm and the removal of false-positive findings were then used to enhance the hepatic boundary more accurately. The liver volume was calculated automatically.

Histologic examination of hepatic steatosis and fat-free liver volume-to-body weight ratio

Donors underwent percutaneous ultrasonographically guided biopsies of the right liver with an 18-gauge needle (Stericut; TSK Laboratory, Tochigi, Japan) on the same day as the CT examination. The degree of hepatic steatosis replacing the hepatic parenchyma was evaluated quantitatively using a percentage scale.

To assess the functioning hepatic mass, excluding the fat, relative to the body size, fat-free liver volumeto-body weight ratio (FFLBWR) was calculated using the following formula: FFLBWR $=$ (estimated liver volume on $\mathrm{CT}$ volumetry $\times[100-$ percentage of 
hepatic steatosis on histologic examination]/100)/body weight.

\section{Indocyanine green test}

The ICG test was performed by rapid intravenous injection of $0.5 \mathrm{mg} / \mathrm{kg}$ body weight of ICG (Dong-in Dang Pharmaceutical Co., Siheong, Korea). Blood samples were drawn from an antecubital vein opposite to the site of injection, before and 15 minutes after ICG administration. After centrifugation, each sample was measured at $805 \mathrm{~nm}$ using a spectrophotometer (Hitachi, Kyoto, Japan). Indocyanine green test retention rate at 15 minutes (ICG R15) was expressed as a percentage of the fluorescent dye retained at 15 minutes.

\section{Statistical analyses}

Descriptive data are shown as means and standard deviations. Spearman correlation coefficients were used to assess the relationships between ICG R15 and PV TAF, FFLBWR, and PV TAF $\times$ FFLBWR. Statistical analyses were performed using IBM SPSS Statistics for Windows, version 21.0 (IBM Corp., Armonk, NY, USA). $P$ values $<.05$ were considered to be statistically significant.

\section{Results}

The means \pm standard deviations and ranges of imaging parameters, including PV diameter, PV TAV, PV TAF, estimated liver volume on CT volumetry, degree of hepatic steatosis, FFLBWR, and ICG 15 values, are summarized in Table 1.

\section{Relationship between portal vein time average flow} and indocyanine green test retention rate at $\mathbf{1 5}$ minutes

Our results showed that PV TAF was weakly correlated with ICG R15 $(\mathrm{r}=-0.375, P<.001)$ (Table 2, Figure 1).

Table 2. Correlations Between Indocyanine Green Test Results and Imaging Parameters

\begin{tabular}{|cccc|}
\hline & PVTAF & FFLBWR & PVTAF $\times$ FFLBWR \\
ICG R15 & $r=-0.375, P<.001$ & $r=-0.239, P<.001$ & $r=-0.424, P<.001$ \\
\hline
\end{tabular}

Abbreviations: FFLBWR, fat-free liver volume-to-body weight ratio; ICG $\mathrm{R} 15$, indocyanine green retention rate at 15 minutes; PV, portal vein; TAF, time average flow
Relationship between fat-free liver volume/body weight ratio and indocyanine green test retention rate at 15 minutes

Our results showed that FFLBWR was weakly correlated with ICG R15 $(r=-0.239, P<.001)$ (Table 2, Figure 2).

Figure 1. Scatter Plots Demonstrating the Relationship Between Portal Vein Time Average Flow and Indocyanine Green Retention Rate at 15 Minutes

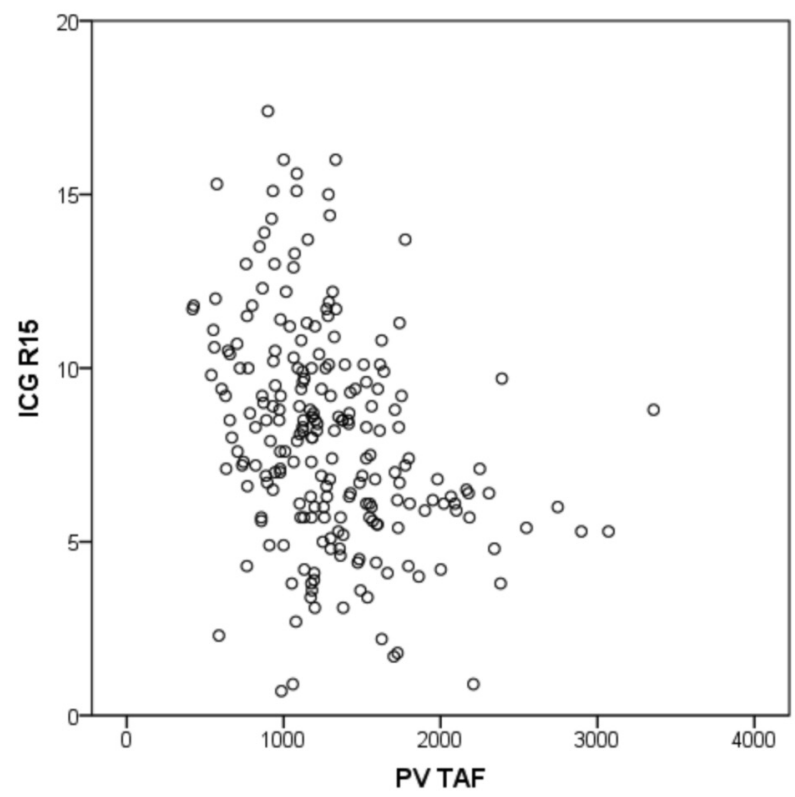

Abbreviations: ICG R15, indocyanine green retention rate at 15 minutes; PV portal vein; TAF, time average flow

Figure 2. Scatter Plots Demonstrating the Relationship Between Fat-Free Live Volume-to-Body Weight Ratio and Indocyanine Green Retention Rate at 15 Minutes

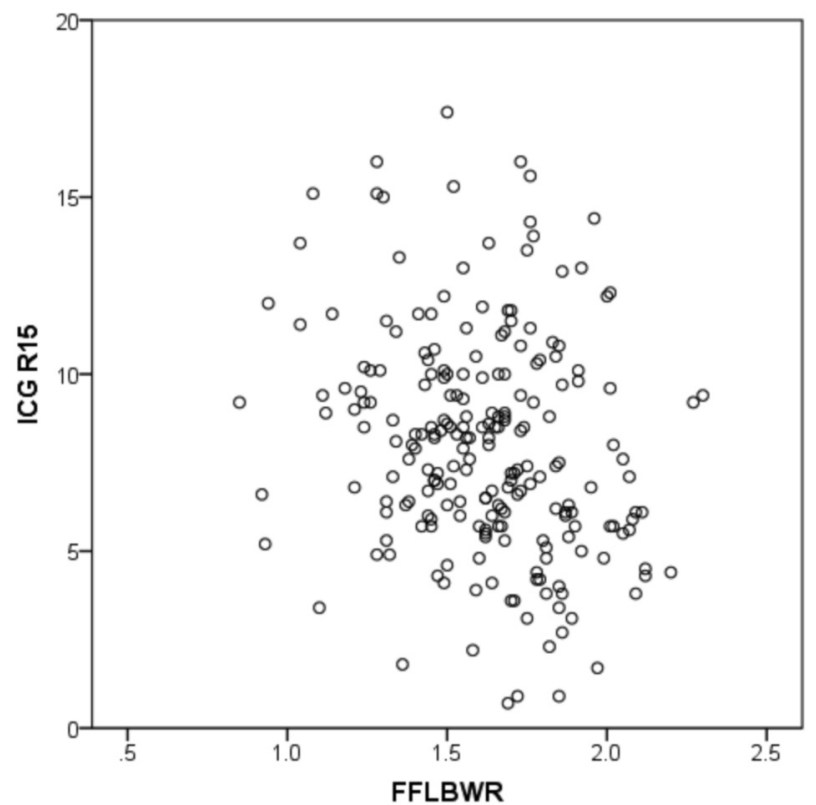

Abbreviations: FFLBWR, fat-free liver volume-to-body weight ratio; ICG $\mathrm{R} 15$, indocyanine green retention rate at 15 minutes 
Relationship between portal vein time average flow $x$ fat-free liver volume-to-body weight ratio and indocyanine green test retention rate at 15 minutes Our results showed that PV TAF $\times$ FFLBWR was moderately correlated with ICG R15 $(r=-0.424$, $P<.001$ ) (Table 2, Figure 3 ).

Figure 3. Scatter Plots Demonstrating the Relationship Between Portal Vein Time Average Flow Multiplied by Fat-Free Liver Volume/Body Weight Ratio and Indocyanine Green Retention Rate at 15 Minutes

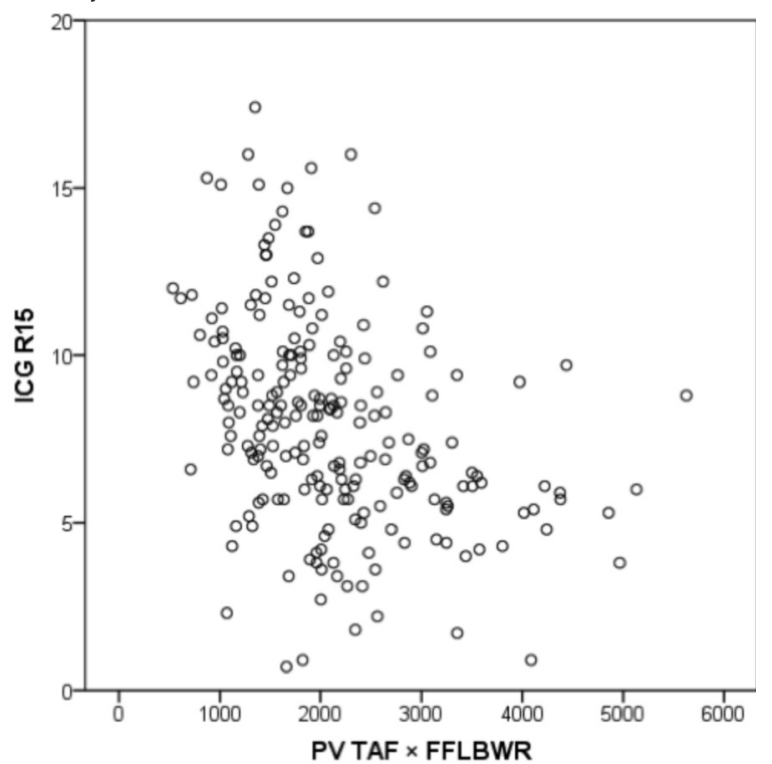

Abbreviations: FFLBWR, fat-free liver volume-to-body weight ratio; ICG $\mathrm{R} 15$, indocyanine green retention rate at 15 minutes; PV, portal vein; TAF, time average flow

\section{Discussion}

We evaluated the correlations between ICG R15 and imaging parameters in living liver donor candidates with well-preserved liver function. In our study, PV TAF, FFLBWR, and PV TAF $\times$ FFLBWR were significantly correlated with ICG R15.

On the basis of animal experimental studies, ${ }^{9,10}$ the ICG test was initially introduced to estimate hepatic blood flow. In a prior study of healthy human subjects, ${ }^{11}$ the ICG test showed the possibility of being used for estimating hepatic blood flow, compared with the previously established method utilizing sulfobromophthalein. Although ICG-based hepatic blood flow measurement has the disadvantage of invasiveness, Doppler ultrasonography allows a noninvasive study of hepatic circulation in real time. ${ }^{5}$ Moreover, in vitro studies have shown that hepatic blood flow measurements by Doppler ultrasonography were accurate and reproducible, ${ }^{4,12}$ and an in vivo study conducted in healthy human subjects reported that Doppler ultrasonography was reliable for estimation of portal blood flow. ${ }^{13}$ In a prior study from Bolognesi and associates ${ }^{14}$ in patients with compensated cirrhosis, an estimation of portal blood flow by Doppler ultrasonography was correlated with ICG-based hepatic blood flow. In our study with a relatively large number of healthy subjects, PV TAF, as surrogate of hepatic blood flow, was significantly correlated with ICG R15, which is consistent with this prior study, ${ }^{14}$ but the correlation was weak.

Another component of the ICG test is liver extraction capacity; notably, functional liver volume reflects liver extraction capacity. Prior studies have reported that liver parenchymal cell volume, measured by $\mathrm{CT}$, and functional liver volume, estimated by magnetic resonance imaging with hepatobiliary-specific contrast agent, showed significant correlations with ICG R15. ${ }^{15,16}$ In our study, we proposed FFLBWR to access functional liver volume in living donor candidates. It is wellknown that donor liver steatosis can reduce the functional hepatic volume. ${ }^{6,17}$ Therefore, we used the FFLBWR as a surrogate of functional liver volume; we observed a significant correlation between FFLBWR and ICG R15, although this correlation was also weak

The ICG retention test is a quantitative test that represents both liver blood flow and functional hepatic reserve. In our study, when we multiplied PV TAF by FFLBWR, this parameter showed moderate correlations with ICG R15 versus the weak correlations shown for both PV TAF and FFLBWR. Therefore, we suggest that PV TAF, as a surrogate of total liver flow, multiplied by FFLBWR, reflecting functional liver reserve in healthy subjects with wellpreserved liver function, improves the correlation with ICG R15.

The ICG retention test is commonly used, especially in the East. However, facilities to measure ICG R15 are not readily available in all countries worldwide. In addition, the ICG test requires additional invasiveness and cost. In contrast, ultrasonography and $\mathrm{CT}$ are routinely used for preoperative evaluations to determine appropriateness of candidates as potential living donors. In this respect, it may be valuable to acknowledge that hepatic blood flow, estimated by Doppler ultrasonography, and functional liver volume, measured by CT volumetry modified according to the degree of steatosis, can reflect the ICG test. We suggest that the correlation 
between imaging parameters and ICG test results may help clinicians decide appropriateness of a candidate as a living liver donor, especially in some centers where facilities to measure ICG R15 are not available.

Our study has some limitations. First, we did not include hepatic arterial flow by Doppler ultrasonography in our measurements of liver blood flow. Total liver blood flow is calculated as the sum of hepatic arterial flow and PV flow. ${ }^{5}$ However, clear visualization of the hepatic artery and Doppler measurements of hepatic arterial flow are possible for only a limited population because of their deep location, where ultrasonography signals are disturbed to a large degree with scatters and clutters; the small size, close to the spatial resolution of the conventional convex transducer; and large variabilities in anatomic configurations of the vessels. ${ }^{4,12}$ In contrast, portal blood flow estimation by Doppler ultrasonography has been proven reliable and reproducible in both healthy subjects and cirrhotic patients. ${ }^{13,14}$ Therefore, we used portal blood flow as a surrogate of liver blood flow. Second, we included only healthy living liver donor candidates; therefore, the results cannot be generalized to patients with liver disease. Third, we did not consider the relationship between the ICG test and static assessments of liver function, including excretory function (total bilirubin) and synthetic function (albumin and prothrombin time). Indocyanine green and bilirubin bind to the same carrier system in hepatocytes; thus, the ICG transport capacity is competitively inhibited in cases of hyperbilirubinemia. ${ }^{1,2}$ However, the patients enrolled in this study were healthy subjects with normal liver function. We suspect that the ICG test solely relied on hepatic clearance, consisting of liver blood flow and liver extraction capacity, in our study population.

In conclusion, imaging parameters, including PV TAF, FFLBWR, and PV TAF $\times$ FFLBWR, were significantly correlated with ICG R15 in healthy subjects recruited from a pool of living liver donor candidates.

\section{References}

1. De Gasperi A, Mazza E, Prosperi M. Indocyanine green kinetics to assess liver function: Ready for a clinical dynamic assessment in major liver surgery? World J Hepatol. 2016;8(7):355-367.

2. Imamura H, Sano K, Sugawara Y, Kokudo N, Makuuchi M. Assessment of hepatic reserve for indication of hepatic resection: decision tree incorporating indocyanine green test. J Hepatobiliary Pancreat Surg. 2005:12(1):16-22.

3. Cherrick GR, Stein SW, Leevy CM, Davidson CS. Indocyanine green: observations on its physical properties, plasma decay, and hepatic extraction. J Clin Invest. 1960;39:592-600.

4. Carlisle KM, Halliwell M, Read AE, Wells PN. Estimation of total hepatic blood flow by duplex ultrasound. Gut. 1992;33(1):92-97.

5. Berzigotti A, Reverter E, Garcia-Criado A, et al. Reliability of the estimation of total hepatic blood flow by Doppler ultrasound in patients with cirrhotic portal hypertension. J Hepatol. 2013;59(4): 717-722.

6. Miller CM, Durand F, Heimbach JK, et al. The International Liver Transplant Society Guideline on Living Liver Donation. Transplantation. 2016;100(6):1238-1243.

7. Lee J, Kim N, Lee H, et al. Efficient liver segmentation using a levelset method with optimal detection of the initial liver boundary from level-set speed images. Comput Methods Programs Biomed. 2007;88(1):26-38

8. Malladi R, Sethian JA, Vemuri BC. Shape modeling with front propagation: a level set approach. IEEE Trans Pattern Anal Machine Intelligence. 1995;17(2):158-175.

9. Teranaka M, Schenk WG. Hepatic blood flow measurement. A comparison of the indocyanine green and electromagnetic techniques normal and abnormal flow states in the dog. Ann Surg. 1977;185(1):58-63.

10. Burczynski FJ, Pushka KL, Sitar DS, Greenway CV. Hepatic plasma flow: accuracy of estimation from bolus injections of indocyanine green. Am J Physiol. 1987;252(5 Pt 2):H953-H962.

11. Leevy CM, Mendenhall CL, Lesko W, Howard MM. Estimation of hepatic blood flow with indocyanine green. J Clin Invest 1962:41:1169-1179.

12. Nakamura T, Moriyasu F, Ban N, et al. Quantitative measurement of abdominal arterial blood flow using image-directed Doppler ultrasonography: superior mesenteric, splenic, and common hepatic arterial blood flow in normal adults. J Clin Ultrasound. 1989;17(4):261-268.

13. Moriyasu F, Ban N, Nishida O, et al. Clinical application of an ultrasonic duplex system in the quantitative measurement of portal blood flow. J Clin Ultrasound. 1986;14(8):579-588.

14. Bolognesi M, Sacerdoti D, Merkel C, Gatta A. Relationship between portal blood flow measured by image-directed Doppler ultrasonography and hepatic blood flow measured by indocyanine green constant infusion in patients with cirrhosis. J Clin Ultrasound. 1995;23(5):297-303.

15. Hashimoto M, Watanabe G. Hepatic parenchymal cell volume and the indocyanine green tolerance test. J Surg Res. 2000;92(2):222227

16. Chang CC, Huang HC, Liu KL, et al. Clinical feasibility of Gd-EOBDTPA-enhanced MR imaging for assessing liver function: validation with ICG tests and parenchymal cell volume. Clin Imaging. 2016;40(4):797-800.

17. Lee SG. A complete treatment of adult living donor liver transplantation: a review of surgical technique and current challenges to expand indication of patients. Am J Transplant 2015;15(1):17-38 